\title{
Evolution of Alfvén waves by three-wave interactions in super-Alfvénic shocks
}

\author{
R. Vainio ${ }^{1}$ and F. Spanier ${ }^{2}$ \\ 1 Department of Physical Sciences, PO Box 64, 00014 University of Helsinki, Finland \\ e-mail: rami.vainio@helsinki.fi \\ ${ }^{2}$ Institut für Theoretische Physik, Lehrstuhl IV: Weltraum- und Astrophysik, Ruhr-Universität Bochum, 44780 Bochum, \\ Germany \\ e-mail: fspanier@tp4.rub.de
}

Received 25 November 2004 / Accepted 24 February 2005

\begin{abstract}
We calculate the evolution of Alfvén waves in super-Alfvénic flows, such as the downstream region of a fast-mode shock, via three-wave interactions between two Aflvén waves and a sound wave. We show that the process has a significant influence on the evolution of the Alfvén wave spectra, driving the system toward a degenerate normalized cross-helicity, $H_{\mathrm{c}} \rightarrow$ \pm 1 . Typical distances in which the system evolves are evaluated, and the effects of the calculated turbulence evolution on testparticle acceleration in super-Alfvénic quasi-parallel shocks are studied. We show that typically the spectrum of accelerated particles is determined by the first-order Fermi acceleration process as described earlier by Vainio \& Schlickeiser (1998, A\&A, 331, 793; 1999, A\&A, 343, 303), and identify some regions of parameter space, where the earlier results are significantly modified either by changes in the predicted scattering-center compression ratio or by stochastic acceleration in the downstream plasma.
\end{abstract}

Key words. acceleration of particles - magnetohydrodynamics (MHD) - plasmas - shock waves - turbulence - waves

\section{Introduction}

The coupling of high-energy particles, such as cosmic-rays, with the space plasma is collisionless, and occurs via resonant wave-particle interactions. Plasma waves carrying magneticfield fluctuations with wavenumbers close to the reciprocal of the particles' Larmor radius can scatter particles efficiently (Skilling 1975a; Schlickeiser 1989a). These scatterings lead to the diffusive propagation of cosmic rays (Skilling 1975a; Schlickeiser 1989b) and are critical for the most widely employed mechanism of particle acceleration, diffusive shock acceleration (Axford et al. 1977; Bell 1978; Blandford \& Ostriker 1978; Krymsky 1977). Low-frequency plasma waves acting as scattering centers tend to isotropize particles in the frame of the waves. Because these scattering centers are converging at the shock, the process leads to acceleration of the particles and to production of a well-known power-law form of the particleenergy spectrum at the shock

$\frac{\mathrm{d} J}{\mathrm{~d} E} \propto p^{-\Gamma} ; \quad \Gamma=\frac{r_{\mathrm{sc}}+2}{r_{\mathrm{sc}}-1}$,

where $E$ and $p$ are the particle energy and momentum,

$r_{\mathrm{sc}}=\frac{V_{1 n}}{V_{2 n}}$

is the compression ratio of the scattering centers, and $V_{1 n}\left(V_{2 n}\right)$ the speed of the scattering centers in the frame of the shock, as measured along the shock normal upstream (downstream) of the shock.

The potential difference between the gas velocity $u$ and the scattering-center velocity $V$ was acknowledged already by Bell (1978): regarding the low-frequency plasma waves responsible for particle scattering as Alfvén waves, the scattering-center speed will be

$V=u+H_{\mathrm{c}} v_{\mathrm{A}}$,

where $v_{\mathrm{A}}$ is the Alfvén speed and $H_{\mathrm{c}}$ the normalized crosshelicity of the scattering Alfvén waves. The latter is related to the intensities of waves propagating parallel $\left(I^{+}\right)$and antiparallel $\left(I^{-}\right)$to the flow direction:

$H_{\mathrm{c}}=\frac{I^{+}-I^{-}}{I^{+}+I^{-}}$

Thus, $H_{\mathrm{c}}=1$ for all waves propagating in the same direction as the flow in the shock frame (i.e., toward the shock in the upstream region and away from the shock in the downstream region), and $H_{\mathrm{c}}=-1$ for anti-parallel propagation. The second case is prevalent in the upstream region of the shock wave, if the scattering waves are produced by the accelerated particles themselves via the streaming instability (Bell 1978).

The flow in fast-mode shock waves is super-Alfvénic throughout, so the upstream fluctuations are carried to the 
shock by the flow, interact with the shock front, and propagate away from the shock towards the far downstream region. Thus, the wave intensities just behind the shock can be calculated from the known shock properties and upstream intensities (McKenzie \& Westphal 1969; Campeanu \& Schlickeiser 1992; Vainio \& Schlickeiser 1998, 1999). In the interaction region, the downstream cross-helicity always assumes a nondegenerate $\left(\left|H_{c 2}\right| \neq 1\right)$ value, no matter what the state of the upstream waves. In low-Mach-number shock waves, however, the downstream cross-helicity is calculated to be $H_{c 2} \approx-1$, because the downstream flow becomes almost Alfvénic and the anti-parallel wave energy is inefficiently transported away from the shock (Vainio \& Schlickeiser 1998, 1999). Since for a parallel shock wave, $u_{2}=u_{1} / r$ and $v_{\mathrm{A} 2}=v_{\mathrm{A} 1} / \sqrt{r}$, where $r$ is the shock's gas compression ratio, we get

$r_{\mathrm{sc}}=\frac{u_{1}-v_{\mathrm{A} 1}}{u_{2}+H_{\mathrm{c} 2} v_{\mathrm{A} 2}}=r \frac{M_{1}-1}{M_{1}+H_{\mathrm{c} 2} \sqrt{r}}$,

where $M_{1}=u_{1} / v_{\mathrm{A} 1}$ is the Alfvénic Mach number of the shock. Note that we have changed the notation from Vainio \& Schlickeiser $(1998,1999)$, so that $M$ now refers to the local Mach number of the flow, whereas in our earlier work, we used $M$ for the Mach number of the shock. Thus, $r_{\mathrm{sc}}$ becomes very large for shock waves approaching the limit $M_{1} \rightarrow \sqrt{r}$ (as simultaneously $u_{2} \rightarrow v_{\mathrm{A} 2}$ and $H_{\mathrm{c} 2} \rightarrow-1$ ).

The canonical power-law spectrum of particles is valid, if we can assume that the extent $L$ of the turbulent region around the shock is large, i.e., if the diffusion length of the accelerated particles fulfills (Ostrowski \& Schlickeiser 1996; Vainio et al. 2000)

$\kappa / V \ll L$

on both sides of the shock. Here, $\kappa=\frac{1}{3} \lambda v$ is the diffusion coefficient of the accelerated particles, and $\lambda$ and $v$ are the scattering mean free path and the particle speed, respectively. In addition, the spectrum of particles will be modified by stochastic acceleration of particles in the downstream region (Ostrowski \& Schlickeiser 1993; Schlickeiser et al. 1993). This means that in order to correctly evaluate the spectrum, we have to consider the evolution of turbulence in the downstream region.

Downstream wave-evolution has been considered before from the point of view of wave-particle interactions treating Alfvén waves and the resonant comic rays as fluids (Ko 1998). The results of these calculations showed that the final state of the system could only include one wave field, if cosmic rays were present. Thus, the model predicted wave fields with degenerate cross-helicity state far downstream. This approach, however, did not cover the possible direct interaction between the wave fields. Therefore, the degenerate cross-helicity could only be achieved through stochastic acceleration of particles, and this acceleration mechanism seemed in many cases even more important than first-order Fermi acceleration at the shock.

In this paper, we will study the evolution of Alfvén waves downstream parallel shocks including the effects of threewave interactions between Alfvén waves and sound waves. Specifically, we employ the model of Chin \& Wentzel (Chin \& Wentzel 1972; Wentzel 1974) to calculate the evolution the
Alfvén waves by assuming the sound waves generated in the interactions are quickly damped by the plasma. We present the result for two extreme cases of the plasma beta, namely (i) $\beta \equiv c_{\mathrm{S}}^{2} / v_{\mathrm{A}}^{2} \ll 1$, which is applicable to weak fast-mode shocks propagating in a cold medium; and (ii) $\beta \gg 1$, which corresponds to the conditions behind a strong shock wave. Here, $c_{\mathrm{S}}$ is the sound speed. In addition to evaluating the steady-state wave spectra as a function of position in the downstream region of a parallel shock, we discuss the implications of our analysis to particle acceleration processes in the shocks.

\section{Evolution of Alfvén wave spectra}

In the present model, Alfvén waves evolve in the downstream region via three-wave interactions, where two Alfvén waves and one sound wave interact. Which of the different possible processes take place depends on the plasma- $\beta$ for which we can distinguish two cases:

- the case of low- $\beta$ plasma $\left(v_{\mathrm{A}}>c_{\mathrm{S}}\right)$ applicable to sub-critical shocks (e.g., in coronal shocks);

- the case of high- $\beta\left(c_{\mathrm{S}}>v_{\mathrm{A}}\right)$ applicable to super-critical shocks.

As these cases involve different physics, we will deal with them in turn.

\subsection{Low $\beta$-plasma}

For the case of a low $\beta$-plasma one Alfvén wave can decay into another Alfvén wave moving in the opposite direction and a sound wave moving in the same direction. Of course, the inverse reaction is also possible

$A^{ \pm} \leftrightarrow A^{\mp}+S^{ \pm}$.

$A$ and $S$ denote Alfvén and sound waves, respectively, and the superscripts denote waves propagating in the forward $\left[X^{+}\right]$ (i.e., away from the shock) and backward $\left[X^{-}\right]$(i.e., toward the shock) direction in the downstream rest frame.

These reactions require that the following resonance conditions are fulfilled

$\omega_{\mathrm{A}}^{ \pm}=\omega_{\mathrm{A}}^{\mp}+\omega_{\mathrm{S}}^{ \pm}$

$k_{\mathrm{A}}^{ \pm}=k_{\mathrm{A}}^{\mp}+k_{\mathrm{S}}^{ \pm}$

with $\omega_{\mathrm{A}}^{ \pm}= \pm k_{\mathrm{A}}^{ \pm} v_{\mathrm{A}}$ and $\omega_{\mathrm{S}}^{ \pm}= \pm k_{\mathrm{S}}^{ \pm} c_{\mathrm{S}}$. A solution with two Alfvén waves propagating in the same direction is not possible, as this would lead to a sound wave with $k_{\mathrm{S}}=0$ (i.e., to no reaction).

Solving the resonance conditions, one can express the properties of two of the waves with the help of the third; for simplicity we will assume the case $k_{\mathrm{A}}^{+} \rightarrow k_{\mathrm{A}}^{-}+k_{\mathrm{S}}^{+}$, and the other case is analogous.

$k_{\mathrm{A}}^{-}=-k_{\mathrm{A}}^{+} \frac{v_{\mathrm{A}}-c_{\mathrm{S}}}{v_{\mathrm{A}}+c_{\mathrm{S}}}$

$k_{\mathrm{S}}^{+}=k_{\mathrm{A}}^{+} \frac{2 v_{\mathrm{A}}}{v_{\mathrm{A}}+c_{\mathrm{S}}}$.

We will now assume two Alfvén wave modes $k_{\mathrm{A}}^{+}$and $k_{\mathrm{A}}^{-}$with occupation numbers $N_{\mathrm{A}}^{+}$and $N_{\mathrm{A}}^{-}$. The evolution of the occupation numbers can be evaluated with the interaction theory developed by Sagdeev \& Galeev (1969). This theory was used by 
Chin \& Wentzel (1972) and Wentzel (1974) to derive equations of the evolution of Alfvén waves, which we will employ. For the present reaction, neglecting spontaneous emission, Chin and Wentzel derived

$$
\begin{aligned}
& \frac{\mathrm{d} N_{\mathrm{A}}^{+}\left(k_{\mathrm{A}}^{+}\right)}{\mathrm{d} t}=-\frac{4 \pi}{v_{\mathrm{A}}+c_{\mathrm{S}}}|V|^{2} \mathfrak{N} \\
& \frac{\mathrm{d} N_{\mathrm{A}}^{-}\left(k_{\mathrm{A}}^{-}\right)}{\mathrm{d} t}=+\frac{4 \pi}{v_{\mathrm{A}}-c_{\mathrm{S}}}|V|^{2} \mathfrak{N} \\
& \frac{\mathrm{d} N_{\mathrm{S}}^{+}\left(k_{\mathrm{S}}^{+}\right)}{\mathrm{d} t}=+\frac{2 \pi}{v_{\mathrm{A}}}|V|^{2} \mathfrak{N}-v_{\mathrm{S}} N_{\mathrm{S}}^{+}\left(k_{\mathrm{S}}^{+}\right)
\end{aligned}
$$

$\mathfrak{N}=N_{\mathrm{A}}^{+}\left(k_{\mathrm{A}}^{+}\right) N_{\mathrm{A}}^{-}\left(k_{\mathrm{A}}^{-}\right)+N_{\mathrm{A}}^{+}\left(k_{\mathrm{A}}^{+}\right) N_{\mathrm{S}}^{+}\left(k_{\mathrm{S}}^{+}\right)-N_{\mathrm{A}}^{-}\left(k_{\mathrm{A}}^{-}\right) N_{\mathrm{S}}^{+}\left(k_{\mathrm{S}}^{+}\right)$

$|V|^{2}=\frac{\omega_{\mathrm{A}}^{+} \omega_{\mathrm{A}}^{-} \omega_{\mathrm{S}}^{+}}{4 \rho c_{\mathrm{S}}^{2}}$

In a first attempt to simplify the foregoing equations, we will analyze the damping of wave modes in our system. It is well known that Alfvén waves are only slightly damped, while sound waves are strongly damped by, e.g., ion viscosity dissipation (Braginskii 1965). Therefore, we will assume that sound waves vanish immediately, i.e., that their damping rate $v_{\mathrm{S}}$ is higher than the rate of their production (related to the threewave interactions). In this case $\mathfrak{N}$ reduces to

$\mathfrak{N} \approx N_{\mathrm{A}}^{+}\left(k_{\mathrm{A}}^{+}\right) N_{\mathrm{A}}^{-}\left(k_{\mathrm{A}}^{-}\right)$

so our system is described completely by

$\frac{\mathrm{d} N_{\mathrm{A}}^{+}\left(k_{\mathrm{A}}^{+}\right)}{\mathrm{d} t}=-\frac{4 \pi}{v_{\mathrm{A}}+c_{\mathrm{S}}}|V|^{2} N_{\mathrm{A}}^{+}\left(k_{\mathrm{A}}^{+}\right) N_{\mathrm{A}}^{-}\left(k_{\mathrm{A}}^{-}\right)$
$\frac{\mathrm{d} N_{\mathrm{A}}^{-}\left(k_{\mathrm{A}}^{-}\right)}{\mathrm{d} t}=+\frac{4 \pi}{v_{\mathrm{A}}-c_{\mathrm{S}}}|V|^{2} N_{\mathrm{A}}^{+}\left(k_{\mathrm{A}}^{+}\right) N_{\mathrm{A}}^{-}\left(k_{\mathrm{A}}^{-}\right)$

$|V|^{2}=\frac{\omega_{\mathrm{A}}^{+} \omega_{\mathrm{A}}^{-} \omega_{\mathrm{S}}^{+}}{4 \rho c_{\mathrm{S}}^{2}}$.

As noted above, Eqs. (17)-(19) deal with only one type of wave interaction: forward-moving Alfvén wave with high $k$ decaying into a lower- $k$ backward-moving Alfvén wave and to a sound wave. But for a complete derivation of the evolution of the Alfvén waves, it is necessary to also include the decay of the backward-moving waves. Thus, the evolution of the forward waves is described by (Chin \& Wentzel 1972)

$\frac{\mathrm{d} N_{\mathrm{A}}^{+}}{\mathrm{d} t}=-\frac{4 \pi}{v_{\mathrm{A}}+c_{\mathrm{S}}}|V|^{2} N_{\mathrm{A}}^{+} N_{\mathrm{A}}^{-}+\frac{4 \pi}{v_{\mathrm{A}}-c_{\mathrm{S}}}\left|V^{*}\right|^{2} N_{\mathrm{A}}^{+} N_{\mathrm{A}}^{-*}$.

Quantities denoted with an asterisk are evaluated at a different $k$, i.e.,

$$
\begin{aligned}
N_{\mathrm{A}}^{-*} & =N_{\mathrm{A}}^{-}\left(k_{\mathrm{A}}^{-*}\right) \\
\left|V^{*}\right|^{2} & =\frac{\omega_{\mathrm{A}}^{+} \omega_{\mathrm{A}}^{-*} \omega_{\mathrm{S}}^{-*}}{4 \rho c_{\mathrm{S}}^{2}} \\
k_{\mathrm{A}}^{-*} & =-k_{\mathrm{A}}^{+} \frac{v_{\mathrm{A}}+c_{\mathrm{S}}}{v_{\mathrm{A}}-c_{\mathrm{S}}} \\
k_{\mathrm{S}}^{-*} & =-k_{\mathrm{A}}^{+} \frac{2 v_{\mathrm{A}}}{v_{\mathrm{A}}-c_{\mathrm{S}}} .
\end{aligned}
$$

Forward waves

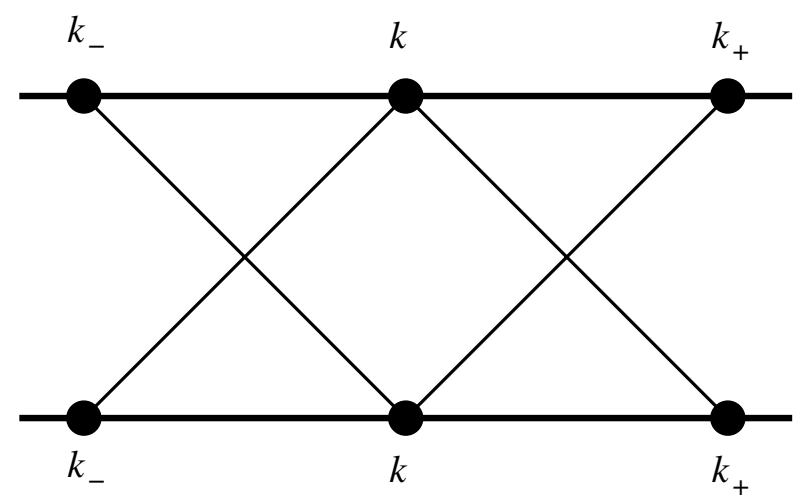

Backward waves

Fig. 1. The Alfvén wave reactions between the wavenumber levels $k$, $k_{+}$and $k_{-}$. The evolution equations and the values of $k_{ \pm}$are given by Eqs. (25)-(28) and (43)-(46) for low and high- $\beta$ plasma, respectively. (Skilling 1975b).

An analogous equation governs the evolution of the backward waves. Simplifying the notation by evaluating the evolution of both Alfvén wave modes at the same $k$ gives

$$
\begin{aligned}
\frac{\mathrm{d} N_{\mathrm{A}}^{+}}{\mathrm{d} t}= & -\frac{4 \pi}{v_{\mathrm{A}}+c_{\mathrm{S}}} \frac{v_{\mathrm{A}} k v_{\mathrm{A}} k_{-} c_{\mathrm{S}}\left(k+k_{-}\right)}{4 \rho c_{\mathrm{S}}^{2}} N_{\mathrm{A}}^{+} N_{\mathrm{A}}^{-}\left(k_{-}\right) \\
& +\frac{4 \pi}{v_{\mathrm{A}}-c_{\mathrm{S}}} \frac{v_{\mathrm{A}} k v_{\mathrm{A}} k_{+} c_{\mathrm{S}}\left(k+k_{+}\right)}{4 \rho c_{\mathrm{S}}^{2}} N_{\mathrm{A}}^{+} N_{\mathrm{A}}^{-}\left(k_{+}\right) \\
\frac{\mathrm{d} N_{\mathrm{A}}^{-}}{\mathrm{d} t}= & +\frac{4 \pi}{v_{\mathrm{A}}-c_{\mathrm{S}}} \frac{v_{\mathrm{A}} k v_{\mathrm{A}} k_{+} c_{\mathrm{S}}\left(k+k_{+}\right)}{4 \rho c_{\mathrm{S}}^{2}} N_{\mathrm{A}}^{+}\left(k_{+}\right) N_{\mathrm{A}}^{-} \\
& -\frac{4 \pi}{v_{\mathrm{A}}+c_{\mathrm{S}}} \frac{v_{\mathrm{A}} k v_{\mathrm{A}} k_{-} c_{\mathrm{S}}\left(k+k_{-}\right)}{4 \rho c_{\mathrm{S}}^{2}} N_{\mathrm{A}}^{+}\left(k_{-}\right) N_{\mathrm{A}}^{-} \\
k_{+}= & \frac{v_{\mathrm{A}}+c_{\mathrm{S}}}{v_{\mathrm{A}}-c_{\mathrm{S}}} k \\
k_{-}= & \frac{v_{\mathrm{A}}-c_{\mathrm{S}}}{v_{\mathrm{A}}+c_{\mathrm{S}}} k,
\end{aligned}
$$

where the wavenumber argument of $N_{\mathrm{A}}$ is omitted when it equals $k$. The reactions between the wavenumber levels are depicted in Fig. 1.

To further simplify these coupled equations we perform a Taylor expansion of terms of the form $k_{ \pm}^{2} N\left(k_{ \pm}\right)$to evaluate all occupation numbers at the same wavenumber:

$k_{ \pm}^{2} N\left(k_{ \pm}\right) \approx k^{2} N(k)+\left(k_{ \pm}-k\right) \frac{\partial\left(k^{2} N\right)}{\partial k}$.

To the lowest order in $\beta$ this results in (Chin \& Wentzel 1972; Skilling 1975b)

$\frac{\mathrm{d} N_{\mathrm{A}}^{+}}{\mathrm{d} t}=8 \pi \frac{k^{2}}{\rho} \frac{\partial\left(k^{2} N_{\mathrm{A}}^{-}\right)}{\partial k} N_{\mathrm{A}}^{+}$
$\frac{\mathrm{d} N_{\mathrm{A}}^{-}}{\mathrm{d} t}=8 \pi \frac{k^{2}}{\rho} \frac{\partial\left(k^{2} N_{\mathrm{A}}^{+}\right)}{\partial k} N_{\mathrm{A}}^{-}$.

We will consider the steady state, in which the time derivatives are given as $\mathrm{d} / \mathrm{d} t=v_{g} \partial / \partial x$, where $v_{g}=u \pm v_{\mathrm{A}}$ is the group 
Table 1. Notation used in the calculations.

\begin{tabular}{ll}
\hline \hline Wave occupation number $\left[\hbar \mathrm{cm}^{-2}\right]$ & $N^{ \pm}$ \\
Wave intensity $\left[\mathrm{G}^{2} \mathrm{~cm}\right]$ & $I^{ \pm}=4 \pi\left|\omega^{ \pm}\right| N^{ \pm}$ \\
Magnetic-field energy density $\left[\mathrm{erg} \mathrm{cm}^{-3}\right]$ & $U_{B}=\frac{1}{2} \rho v_{\mathrm{A}}^{2}$ \\
Dimensionless wave-energy density & $\mathfrak{E}^{ \pm}=k\left|\omega^{ \pm}\right| N^{ \pm} / U_{B}$ \\
Logarithmic wavenumber & $y=\ln \left(k / k_{0}\right)$ \\
Dimensionless distance & $x^{\prime}=k_{0} x$ \\
Unit of $k$ (arbitrary) $\left[\mathrm{cm}^{-1}\right]$ & $k_{0}$ \\
Plasma beta & $\beta=c_{\mathrm{S}}^{2} / v_{\mathrm{A}}^{2}$ \\
Local Mach number & $M=u / v_{\mathrm{A}}$ \\
\hline
\end{tabular}

velocity of the waves and $x$ distance from the shock. Thus,

$$
\begin{aligned}
& \left(u+v_{\mathrm{A}}\right) \frac{\partial N_{\mathrm{A}}^{+}}{\partial x}=8 \pi \frac{k^{2}}{\rho} \frac{\partial\left(k^{2} N_{\mathrm{A}}^{-}\right)}{\partial k} N_{\mathrm{A}}^{+} \\
& \left(u-v_{\mathrm{A}}\right) \frac{\partial N_{\mathrm{A}}^{-}}{\partial x}=8 \pi \frac{k^{2}}{\rho} \frac{\partial\left(k^{2} N_{\mathrm{A}}^{+}\right)}{\partial k} N_{\mathrm{A}}^{-}
\end{aligned}
$$

We will now introduce a dimensionless notation summarized in Table 1. This involves the use of wave-energy densities normalized to the background magnetic field energy density, $\mathfrak{E}^{ \pm}=k\left|\omega^{ \pm}\right| N_{\mathrm{A}}^{ \pm} / U_{B}$. Thus, Eqs. (32) and (33) yield

$$
\frac{\partial \mathfrak{E}^{ \pm}}{\partial x}=\frac{4 \pi k^{2}}{M \pm 1} \frac{\partial \mathfrak{E}^{\mp}}{\partial k} \mathfrak{E}^{ \pm} \text {. }
$$

Further, introducing a logarithmic wavenumber, $y=\ln \left(k / k_{0}\right)$ and a dimensionless distance, $x^{\prime}=k_{0} x$, normalized to the arbitrary unit of wavenumber, $k_{0}$, we get

$$
\frac{\partial \ln \mathfrak{E}^{ \pm}}{\partial x^{\prime}}=\frac{4 \pi \mathrm{e}^{y}}{M \pm 1} \frac{\partial \mathfrak{E}^{\mp}}{\partial y} \text {. }
$$

Although the set of equations is compact, we have found no general analytical solution for it. Analytical results in some special cases, as well as numerical results, are given in the results section. Using the dimensionless notation in Eqs. (25)(26) results in

$\frac{\partial \ln \mathfrak{E}^{ \pm}}{\partial x^{\prime}}=\frac{4 \pi \mathrm{e}^{y}}{M \pm 1} \frac{\mathfrak{E}^{\mp}\left(y_{+}\right)-\mathfrak{E}^{\mp}\left(y_{-}\right)}{4 \sqrt{\beta}(1-\beta)}$,

where

$$
\begin{aligned}
& y_{ \pm}=\ln \frac{k_{ \pm}}{k_{0}}=y \pm \Delta y \\
& \Delta y=\ln \frac{v_{\mathrm{A}}+c_{\mathrm{S}}}{v_{\mathrm{A}}-c_{\mathrm{S}}} \approx 2 \sqrt{\beta} .
\end{aligned}
$$

Thus, the numerical solution is accurately obtained using a uniform $y$-grid with spacing $\Delta y$ given by Eq. (38). We also use a uniform grid for $x^{\prime}$, and a simple explicit method for the spatial differencing.

\subsection{High $\beta$-plasma}

For the case of a high $\beta$-plasma two oppositely moving Alfvén waves can decay into a sound wave moving in the direction of the Alfvén wave with higher $k$ :

$$
\begin{array}{ll}
A^{+}+A^{-} \leftrightarrow S^{+} & \left(\left|k_{\mathrm{A}}^{+}\right|>\left|k_{\mathrm{A}}^{-}\right|\right) \\
A^{+}+A^{-} \leftrightarrow S^{-} & \left(\left|k_{\mathrm{A}}^{+}\right|<\left|k_{\mathrm{A}}^{-}\right|\right) .
\end{array}
$$

As in the case of low- $\beta$ plasma we will analyze the first reaction only to derive the basic equation, and from there on, we will add the second reaction to derive the full equation for the Alfvén wave evolution. For the first reaction (Chin \& Wentzel 1972; Wentzel 1974)

$$
\begin{aligned}
& \frac{\mathrm{d} N_{\mathrm{A}}^{ \pm}\left(k_{\mathrm{A}}^{ \pm}\right)}{\mathrm{d} t}=\frac{4 \pi}{v_{\mathrm{A}}+c_{\mathrm{S}}}|V|^{2} \mathfrak{N} \\
& \frac{\mathrm{d} N_{\mathrm{S}}^{+}\left(k_{\mathrm{S}}^{+}\right)}{\mathrm{d} t}=-\frac{2 \pi}{v_{\mathrm{A}}}|V|^{2} \mathfrak{N}-v_{\mathrm{S}} N_{\mathrm{S}}^{+}\left(k_{\mathrm{S}}^{+}\right)
\end{aligned}
$$

$\mathfrak{N}=N_{\mathrm{A}}^{-}\left(k_{\mathrm{A}}^{-}\right) N_{\mathrm{S}}^{+}\left(k_{\mathrm{S}}^{+}\right)+N_{\mathrm{A}}^{+}\left(k_{\mathrm{A}}^{+}\right) N_{\mathrm{S}}^{+}\left(k_{\mathrm{S}}^{+}\right)-N_{\mathrm{A}}^{+}\left(k_{\mathrm{A}}^{+}\right) N_{\mathrm{A}}^{-}\left(k_{\mathrm{A}}^{-}\right)$

$|V|^{2}=\frac{\omega_{\mathrm{A}}^{+} \omega_{\mathrm{A}}^{-} \omega_{\mathrm{S}}^{+}}{4 \rho c_{\mathrm{S}}^{2}}$

Our basic assumption is the same as before: the damping of the sound waves is strong enough to dissipate the waves immediately, which makes all $N_{\mathrm{S}}^{+}$terms negligible. Including the second reaction and proceeding similarly as in the low- $\beta$ case, we can write

$$
\begin{aligned}
\frac{\mathrm{d} N_{\mathrm{A}}^{+}}{\mathrm{d} t}= & -\frac{4 \pi}{v_{\mathrm{A}}+c_{\mathrm{S}}} \frac{v_{\mathrm{A}} k v_{\mathrm{A}} k_{+} c_{\mathrm{S}}\left(k_{+}-k\right)}{4 \rho c_{\mathrm{S}}^{2}} N_{\mathrm{A}}^{+} N_{\mathrm{A}}^{-}\left(k_{+}\right) \\
& -\frac{4 \pi}{v_{\mathrm{A}}+c_{\mathrm{S}}} \frac{v_{\mathrm{A}} k v_{\mathrm{A}} k_{-} c_{\mathrm{S}}\left(k-k_{-}\right)}{4 \rho c_{\mathrm{S}}^{2}} N_{\mathrm{A}}^{+} N_{\mathrm{A}}^{-}\left(k_{-}\right) \\
\frac{\mathrm{d} N_{\mathrm{A}}^{-}}{\mathrm{d} t}= & -\frac{4 \pi}{v_{\mathrm{A}}+c_{\mathrm{S}}} \frac{v_{\mathrm{A}} k v_{\mathrm{A}} k_{+} c_{\mathrm{S}}\left(k_{+}-k\right)}{4 \rho c_{\mathrm{S}}^{2}} N_{\mathrm{A}}^{+}\left(k_{+}\right) N_{\mathrm{A}}^{-} \\
& -\frac{4 \pi}{v_{\mathrm{A}}+c_{\mathrm{S}}} \frac{v_{\mathrm{A}} k v_{\mathrm{A}} k_{-} c_{\mathrm{S}}\left(k-k_{-}\right)}{4 \rho c_{\mathrm{S}}^{2}} N_{\mathrm{A}}^{+}\left(k_{-}\right) N_{\mathrm{A}}^{-} .
\end{aligned}
$$

Here, the occupation numbers are again evaluated at $k$ unless otherwise indicated, and $k_{+}$and $k_{-}$again denote the higher and lower wavenumber levels participating in the reactions, as described in Fig. 1. However, the values of these wavenumbers are now

$k_{+}=\frac{c_{\mathrm{S}}+v_{\mathrm{A}}}{c_{\mathrm{S}}-v_{\mathrm{A}}} k$
$k_{-}=\frac{c_{\mathrm{S}}-v_{\mathrm{A}}}{c_{\mathrm{S}}+v_{\mathrm{A}}} k$.

Now $k_{ \pm}^{2} N\left(k_{ \pm}\right)$can again be Taylor-expanded using Eq. (29) and inserting these results in Eqs. (43) and (44), the derivatives of $k^{2} N$ with respect to $k$ exactly cancel. Thus,

$$
\begin{aligned}
\frac{\mathrm{d} N_{\mathrm{A}}^{+}}{\mathrm{d} t} & =\left(u+v_{\mathrm{A}}\right) \frac{\partial N_{\mathrm{A}}^{+}}{\partial x} \\
& =-\frac{4 \pi}{v_{\mathrm{A}}+c_{\mathrm{S}}} \frac{v_{\mathrm{A}}^{3} k^{3}}{\rho\left(c_{\mathrm{S}}^{2}-v_{\mathrm{A}}^{2}\right)} N_{\mathrm{A}}^{+} N_{\mathrm{A}}^{-} \\
\frac{\mathrm{d} N_{\mathrm{A}}^{-}}{\mathrm{d} t} & =\left(u-v_{\mathrm{A}}\right) \frac{\partial N_{\mathrm{A}}^{-}}{\partial x} \\
& =-\frac{4 \pi}{v_{\mathrm{A}}+c_{\mathrm{S}}} \frac{v_{\mathrm{A}}^{3} k^{3}}{\rho\left(c_{\mathrm{S}}^{2}-v_{\mathrm{A}}^{2}\right)} N_{\mathrm{A}}^{+} N_{\mathrm{A}}^{-},
\end{aligned}
$$

where all occupation numbers are evaluated at $k$, which now appears only as a parameter in these equations. (Thus, $\partial / \partial x \rightarrow$ $\mathrm{d} / \mathrm{d} x$.) We will again make use of the dimensionless quantities 
$\mathfrak{E}^{ \pm}=k\left|\omega^{ \pm}\right| N_{\mathrm{A}}^{ \pm} / U_{B}$ and denote

$k_{1}=\frac{2 \pi v_{\mathrm{A}}^{3} k}{\left(c_{\mathrm{S}}^{2}-v_{\mathrm{A}}^{2}\right)\left(c_{\mathrm{S}}+v_{\mathrm{A}}\right)}=k \frac{2 \pi}{(\beta-1)(\sqrt{\beta}+1)}$

$\frac{\mathrm{d} \mathfrak{E}^{ \pm}}{\mathrm{d} x}=-k_{1} \frac{\mathfrak{E}^{+} \mathfrak{E}^{-}}{M \pm 1}$

This gives a dimensionless distance (now wavenumber dependent) as $x^{\prime}=k_{1} x$ and, finally,

$\frac{\mathrm{d} \mathfrak{E}^{ \pm}}{\mathrm{d} x^{\prime}}=-\frac{\mathfrak{E}^{+} \mathfrak{E}^{-}}{M \pm 1}$

These coupled ordinary differential equations may be solved analytically with boundary conditions at the shock $\left(x^{\prime}=0\right)$,

$\mathfrak{E}^{ \pm}(0 ; k)=\mathfrak{E}_{0}^{ \pm}(k)$,

as

$$
\begin{aligned}
\mathfrak{E}^{+} & =\frac{(M+1) \mathfrak{E}_{0}^{+}-(M-1) \mathfrak{E}_{0}^{-}}{(M+1) \mathfrak{E}_{0}^{+}-(M-1) \mathfrak{E}_{0}^{-} \exp \left\{-g x^{\prime}\right\}} \mathfrak{E}_{0}^{+} \\
\mathfrak{E}^{-} & =\frac{(M+1) \mathfrak{E}_{0}^{+}-(M-1) \mathfrak{E}_{0}^{-}}{(M+1) \mathfrak{E}_{0}^{+} \exp \left\{+g x^{\prime}\right\}-(M-1) \mathfrak{E}_{0}^{-}} \mathfrak{E}_{0}^{-} \\
g & =\frac{\mathfrak{E}_{0}^{+}}{M-1}-\frac{\mathfrak{E}_{0}^{-}}{M+1} .
\end{aligned}
$$

Note that

$\frac{\mathfrak{E}^{+}\left(x^{\prime} ; k\right)}{M-1}-\frac{\mathfrak{E}^{-}\left(x^{\prime} ; k\right)}{M+1}=g \quad \forall x^{\prime} \geq 0$.

The properties of the solution will be studied in the next section.

\section{Results and discussion}

\subsection{Low $\beta$-plasma}

Since we had not found a general analytical solution in the case of the low $\beta$-plasma, we developed a numerical approach. However, some special solutions can be found analytically, which we will present first.

\subsubsection{Analytical solutions}

Below, we list some useful analytical solutions we found for the case of low- $\beta$ plasma.

1. Two equilibrium (i.e., $\partial \mathfrak{F}^{ \pm} / \partial x=0$ ) solutions:

- an equilibrium solution is obtained when either $\mathfrak{E}^{+}$or $\mathfrak{E}^{-}$is zero. In this case, no three-wave interactions can take place;

- another equilibrium solution is obtained by setting both $\mathfrak{F}^{ \pm}$constants. Three-wave interactions still transport the energy from higher to lower values of $k$ maintaining stationary wave spectra. This, however, implies an infinite total wave energy density.
2. A separable solution:

if $\mathfrak{E}^{ \pm}$are assumed to be separable in $x$ and $k$, i.e., $\mathfrak{E}^{ \pm}=$ $\mathfrak{R}^{ \pm}(k) \mathfrak{X}^{ \pm}(x)$, we get

$\frac{1}{\mathfrak{X}^{+} \mathfrak{X}^{-}} \frac{d \mathfrak{X}^{ \pm}}{d x}=\frac{4 \pi k^{2}}{M \pm 1} \frac{d \mathfrak{\Re}^{\mp}}{d k} \equiv-\frac{k_{0}^{ \pm}}{M \pm 1}$,

where $k_{0}^{ \pm}$are constants. Thus, assuming $\Omega^{ \pm} \rightarrow 0$ as $k \rightarrow \infty$,

$\Omega^{ \pm}=\frac{k_{0}^{\mp}}{4 \pi k}$.

This implies an infinite total wave-energy density, as well. Therefore

$k \frac{\partial \mathfrak{k}^{ \pm}}{\partial k}=-\mathfrak{E}^{ \pm}$

and

$\frac{\partial \mathfrak{F}^{ \pm}}{\partial x}=-\frac{4 \pi k}{M \pm 1} \mathfrak{E}^{-} \mathfrak{E}^{+}$.

Denoting $x^{\prime}=4 \pi k x$, we can read the full solution from Eqs. (53)-(55).

3. A constant cross-helicity solution:

if

$\frac{\mathfrak{E}_{0}^{+}(k)}{M-1}=\frac{\mathfrak{E}_{0}^{-}(k)}{M+1} \equiv \mathfrak{E}_{0}(k) \quad \forall k$,

the ratio of forward and backward propagating waves remains constant at all wavenumbers, no matter what the form of the spectrum is. Thus,

$\mathfrak{E}=\frac{\mathfrak{E}^{+}}{M-1}=\frac{\mathfrak{E}^{-}}{M+1}$

is governed by

$\frac{\partial \mathfrak{E}}{\partial x}=4 \pi k^{2} \mathfrak{E} \frac{\partial \mathfrak{E}}{\partial k}$,

which can be written as

$\frac{\partial \mathfrak{E}}{\partial x}+\mathfrak{E} \frac{\partial \mathfrak{E}}{\partial \ell}=0 ; \ell=1 /(4 \pi k)$

with the formal solution

$\mathfrak{E}(x, \ell)=\mathfrak{E}_{0}(\ell-\mathfrak{E}(x, \ell) x) ; \quad \mathfrak{E}_{0}(\ell)=\mathfrak{E}(0, \ell)$,

or changing back to $k$,

$\mathfrak{E}(x, k)=\mathfrak{E}_{0}\left(\frac{k}{1-4 \pi \mathfrak{E}(x, k) x k}\right) ; \quad \mathfrak{E}_{0}(k)=\mathfrak{E}(0, k)$.

Whether this can be solved explicitly for $\mathbb{E}$ depends on the form of the initial spectrum $\tilde{E}_{0}(k)$. In any case, an upper limit for the spectrum can be obtained as

$\mathfrak{E}(x, k)<\frac{1}{4 \pi k x}=\mathfrak{E}_{\infty}(x, k) \quad \forall x, k>0$,

with $\tilde{E}_{\infty}$ the asymptotic form of the spectrum at large $k x$.

4. A large cross-helicity solution:

assuming that

$\frac{\mathfrak{E}_{0}^{+}}{M-1} \gg \frac{\mathfrak{E}_{0}^{-}}{M+1}$ and $\frac{\partial \mathfrak{E}_{0}^{+}}{\partial k}<0$, 
the effect of the cascade on $\mathfrak{F}^{+}$can be neglected (and simi-

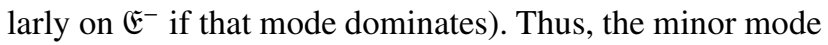
is governed by a linear equation:

$\frac{\partial \mathfrak{E}^{\mp}}{\partial x}=\frac{4 \pi k^{2}}{M \mp 1} \mathfrak{E}^{\mp} \frac{\partial \mathfrak{E}_{0}^{ \pm}}{\partial k}$,

yielding

$\mathfrak{E}^{ \pm}(x, k) \approx \mathfrak{E}_{0}^{ \pm}(k)$

$\mathfrak{E}^{\mp}(x, k) \approx \mathfrak{E}_{0}^{\mp}(k) \exp \left(\frac{4 \pi k^{2}}{M \mp 1} \frac{\partial \mathfrak{E}_{0}^{ \pm}}{\partial k} x\right)$,

$$
\text { if } \quad \frac{\mathfrak{E}_{0}^{ \pm}}{M \mp 1} \gg \frac{\mathfrak{E}_{0}^{\mp}}{M \pm 1} \text { and } \frac{\partial \mathfrak{E}_{0}^{ \pm}}{\partial k}<0 \text {. }
$$

\subsubsection{Numerical solutions}

We have numerically evaluated the behavior of the solutions for three different cases corresponding to

$\frac{\mathfrak{E}_{0}^{+}}{\mathfrak{E}_{0}^{-}}<\frac{M-1}{M+1}, \frac{\mathfrak{E}_{0}^{+}}{\mathfrak{E}_{0}^{-}}=\frac{M-1}{M+1}, \frac{\mathfrak{E}_{0}^{+}}{\mathfrak{E}_{0}^{-}}>\frac{M-1}{M+1}$.

Specifically, as initial configuration we have chosen a broken power law of the form

$\mathfrak{E}_{0}^{ \pm}(k)=C^{ \pm} \frac{10^{\alpha / 2}}{\left(10^{\alpha}+k / k_{0}\right)^{1 / 2}}, \quad k>k_{0}$,

where $k_{0}$ is the lowest wavenumber in the spectrum, and $\alpha$ and $C^{ \pm}$are constants. In the following, we use the values $\alpha=3$, $C^{-}=1$, and

$$
\begin{cases}\text { Case A: } & C^{+}=\frac{M-1}{M+1} \\ \text { Case B: } & C^{+}=e^{-0.1} \frac{M-1}{M+1} \\ \text { Case C: } & C^{+}=e^{+0.1} \frac{M-1}{M+1} .\end{cases}
$$

As a boundary condition we use $\partial^{2}(\ln \mathfrak{E}) / \partial y^{2}=0$ at the lowest and the highest $y$-levels, $y=0$ and $y=10 \ln 10$, on the grid. The three cases are plotted for $M=1.5$ in Figs. 2-4, respectively.

Figures 2-4 show that the evolution of $H_{\mathrm{c}}(k)$ depends on the sign of $g$ (Eq. (55)). When $g$ is zero (Fig. 2) the cross-helicity maintains its constant value, $H_{\mathrm{c}}=-M^{-1}$, at all distances and wavenumbers. The spectra, being flatter than $\mathfrak{E}^{ \pm} \propto k^{-1}$ at low wavenumbers, steepen at high wavenumbers and tend toward the asymptotic spectrum given by Eq. (67). This steepening is transported toward lower wavenumbers as $x$ increases.

If $g$ is different from zero, one of the waves will be damped completely, i.e., the steepening does not stop at the asymptotic level; but the minor-mode spectrum tends to zero even faster at high $k$. For a positive sign of $g$ the backward waves are damped (Fig. 4) and vice versa (Fig. 3), i.e., $H_{\mathrm{c}} \rightarrow \operatorname{sgn} g$ as $x k \rightarrow \infty$. The spectral index of the remaining mode tends toward its initial value. More complicated behavior can be expected in case $g$ is $k$-dependent.

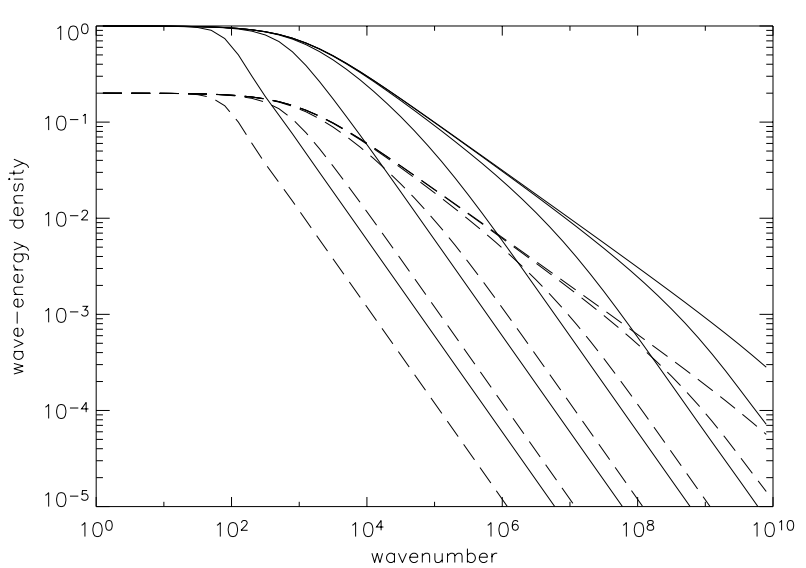

Fig. 2. Wave-energy densities $\mathfrak{E}^{+}$(dashed line) and $\mathfrak{E}^{-}$(solid line) at different positions $x_{i} k_{0}=10^{-8+i}\{i=0,1, \ldots, 5\}$. The parameters are $\beta=0.05$ and $M=1.5$. The cross-helicity has the critical value $H_{\mathrm{c}}=$ $-M^{-1} \Rightarrow \mathfrak{E}^{+} / \mathfrak{E}^{-}=(M-1) /(M+1)$ (i.e., $\left.g=0\right)$.

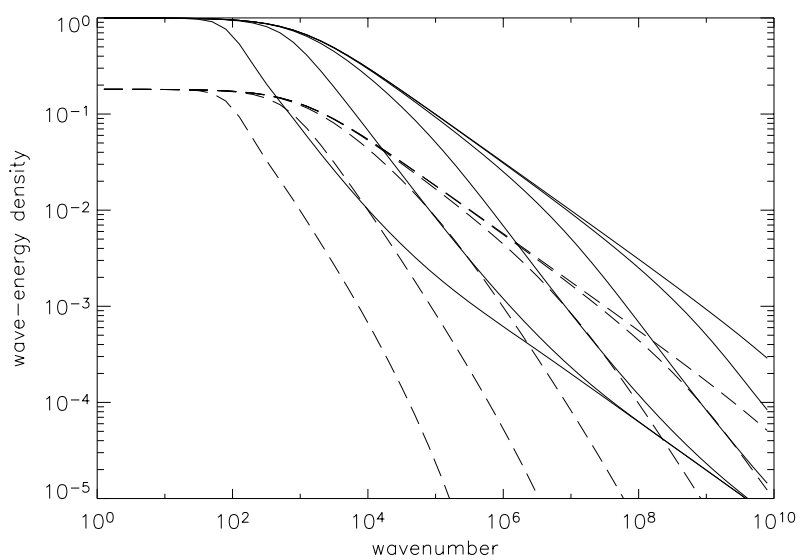

Fig. 3. As Fig. 2, but $\mathfrak{E}^{+} / \mathfrak{E}^{-}=(M-1) e^{-0.1} /(M+1)$ (i.e., $\left.g<0\right)$.

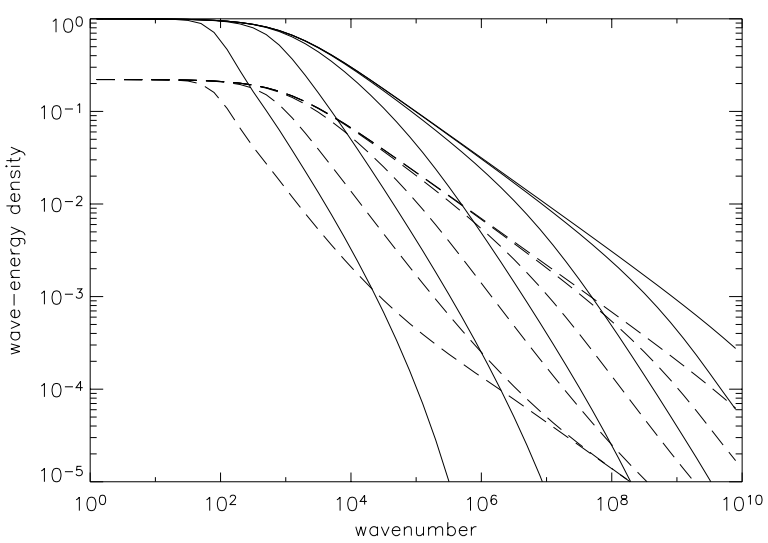

Fig. 4. As Fig. 2, but $\mathfrak{E}^{+} / \mathfrak{E}^{-}=(M-1) e^{+0.1} /(M+1)$ (i.e., $\left.g>0\right)$.

\subsection{High $\beta$-plasma}

With our given analytical results from Eqs. (53) and (54) we can distinguish several special cases. These cases again depend on the sign of $g$. 


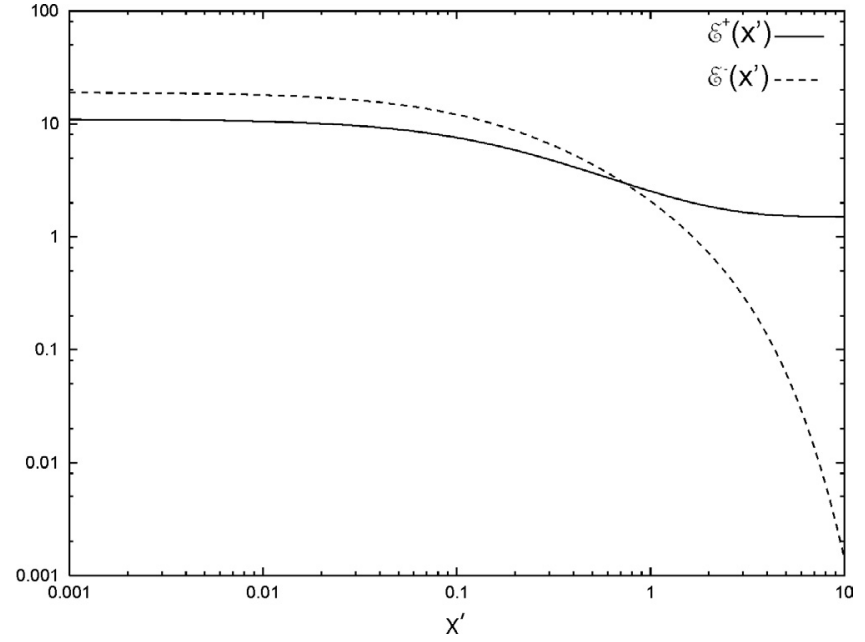

Fig. 5. $\mathfrak{E}^{+}(x)$ and $\mathfrak{E}^{-}(x)$ vs $x^{\prime}$ for $g>0$.

- If $g=0$, the ratio of forward and backward waves (or the cross-helicity $H_{\mathrm{c}}$ ) is constant. The solution in this case is written as

$\mathfrak{E}^{ \pm}=(M \mp 1) \frac{\mathfrak{E}_{0}(k)}{1+\mathfrak{E}_{0}(k) x k_{1}}$,

where $\tilde{E}_{0}(k)$ gives the form of the initial spectrum and $k_{1}(k)$ is given by Eq. (49).

- If $g>0$, we get the following limits for $x^{\prime} \rightarrow \infty$

$$
\begin{aligned}
& \mathfrak{E}^{+}\left(x^{\prime} \rightarrow \infty\right)=\mathfrak{E}_{0}^{+}\left(1-\frac{M-1}{M+1} \frac{\mathfrak{E}_{0}^{-}}{\mathfrak{E}_{0}^{+}}\right) \\
& \mathfrak{E}^{-}\left(x^{\prime} \rightarrow \infty\right)=0 .
\end{aligned}
$$

- If $g<0$, we get

$$
\begin{aligned}
& \mathfrak{E}^{+}\left(x^{\prime} \rightarrow \infty\right)=0 \\
& \mathfrak{E}^{-}\left(x^{\prime} \rightarrow \infty\right)=\mathfrak{E}_{0}^{-}\left(1-\frac{M+1}{M-1} \frac{\mathfrak{E}_{0}^{+}}{\mathfrak{E}_{0}^{-}}\right) .
\end{aligned}
$$

Full solutions for the two latter cases $(g \neq 0)$ are plotted in Figs. 5 and 6. For these cases, we can give the typical damping length $d$ in which the minor Alfvén waves are dissipated and the cross-helicity assumes a degenerate value, $H_{\mathrm{c}} \rightarrow \operatorname{sgn} g$, as

$$
\begin{aligned}
\frac{1}{d} & =\left|g k_{1}\right|=k \frac{2 \pi v_{\mathrm{A}}^{3}}{\left(c_{\mathrm{S}}^{2}-v_{\mathrm{A}}^{2}\right)\left(c_{\mathrm{S}}+v_{\mathrm{A}}\right)}\left|\frac{\mathfrak{E}_{0}^{-}}{M+1}-\frac{\mathfrak{E}_{0}^{+}}{M-1}\right| \\
& =k \frac{2 \pi \mid M H_{\mathrm{c} 0+1 \mid}}{(\beta-1)(\sqrt{\beta}+1)\left(M^{2}-1\right)}\left(\mathfrak{E}_{0}^{-}+\mathfrak{E}_{0}^{+}\right),
\end{aligned}
$$

where $H_{\mathrm{c} 0}$ is the cross-helicity at the shock, $x=0$. The value of $d$ is important for particle acceleration at shock waves, as discussed in the next section.

\subsection{Particle acceleration at shocks}

Apart from the special case of $g=0$, i.e., $H_{\mathrm{c}}=-M^{-1}$, threewave interactions studied in this paper are not able to remove all power from Alfvén waves. Thus, the turbulent region downstream a shock wave may extend very far from the shock, and the first-order Fermi process accelerates particles efficiently.

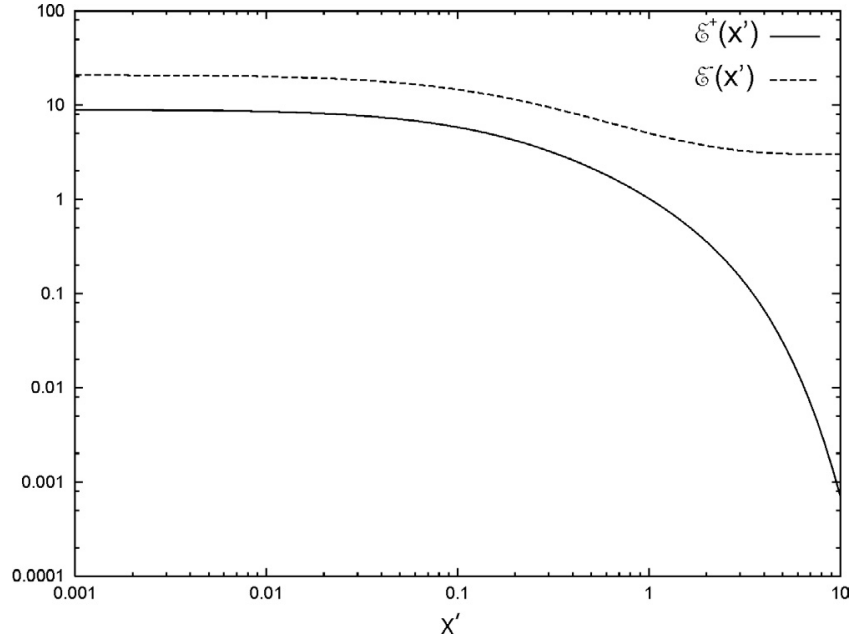

Fig. 6. $\mathfrak{E}^{+}(x)$ and $\mathfrak{E}^{-}(x)$ vs $x^{\prime}$ for $g<0$.

The mean free path of the energetic particles in an Alfvén-wave field with dimensionless energy densities $\mathfrak{E}^{ \pm}(k)$ is (Skilling 1975c)

$\lambda \approx \frac{4}{\pi k} \frac{1}{\mathfrak{E}^{+}(k)+\mathfrak{E}^{-}(k)}$,

where $k=r_{\mathrm{L}}^{-1}$ is the Larmor radius of the particle. Thus, for $\beta>1$,

$d \approx \lambda_{x=0} \frac{(\beta-1)(\sqrt{\beta}+1)\left(M^{2}-1\right)}{8\left|M H_{\mathrm{c} 0}+1\right|}$,

and for $\beta \ll 1$ and $\mathfrak{E}^{ \pm} \propto k^{-1}$

$d \approx \lambda_{x=0} \frac{M^{2}-1}{16\left|M H_{\mathrm{c} 0}+1\right|}$.

Apart from a factor of the order unity, the result is the same for other spectral forms with $\partial \mathfrak{E} / \partial k<0$. The ratio

$$
\begin{aligned}
R_{\mathrm{I}} & =\frac{V d}{\kappa_{x=0}}=\frac{3 V d}{v \lambda_{x=0}} \\
& \approx \begin{cases}\frac{3 \beta^{3 / 2}\left(M^{2}-1\right)\left(M+H_{\mathrm{c} 0}\right)}{8\left|M H_{\mathrm{c} 0}+1\right|} \frac{v_{\mathrm{A}}}{v} & \beta \gg 1 \\
\frac{3\left(M^{2}-1\right)\left(M+H_{\mathrm{c} 0}\right)}{16\left|M H_{\mathrm{c} 0}+1\right|} \frac{v_{\mathrm{A}}}{v} & \beta \ll 1\end{cases}
\end{aligned}
$$

determines the distance over which the waves attain their final cross-helicity in the units of diffusion length of the particles. If $R_{\mathrm{I}} \gg 1$, the spectral index can be determined from the scattering-center compression ratio measured at the shock, like in Vainio \& Schlickeiser $(1998,1999)$. On the other hand, for $R_{\mathrm{I}} \ll 1$ the spectral index of the accelerated particles is determined by the scattering-center compression ratio calculated from the mode remaining in the system, i.e.,

$r_{\mathrm{sc}}=r \frac{M_{1}-1}{M_{1}+\operatorname{sgn} g \sqrt{r}}$

Evidently, if $R_{\mathrm{I}} \ll 1$ and $g \rightarrow 0+$, the results of the analysis of Vainio \& Schlickeiser $(1998,1999)$ will be heavily 
modified, and the flat particle spectra will be modified toward much softer behavior. The wave-transmission model of Vainio \& Schlickeiser $(1998,1999)$ typically predicts $g<0$ for low-Mach-number shocks consistent with the predicted hard particle-energy spectra. However, if the upstream wave amplitude increases as a result of efficient particle acceleration (through the strong streaming instability), cross-helicities at the shock tend to be closer to zero (Vainio \& Schlickeiser 1999). Thus, the present model may offer a quenching mechanism for the shock to avoid the diverging particle pressures plaguing the original model of Vainio \& Schlickeiser (1998).

Stochastic acceleration in the downstream region has the time scale $\tau_{\mathrm{II}}=p^{2} / D$, where (Skilling 1975c)

$D \approx \frac{1-H_{\mathrm{c}}^{2}}{3} p^{2} \frac{v_{\mathrm{A}}^{2}}{v \lambda}$

is the momentum diffusion coefficient. The importance of stochastic acceleration in the downstream region is, therefore, determined by

$R_{\mathrm{II}}=\int_{0}^{\infty} \frac{\mathrm{d} x}{V \tau_{\mathrm{II}}}=\int_{0}^{\infty} \frac{D \mathrm{~d} x}{p^{2} V}$.

If $R_{\mathrm{II}}(p)>1$, stochastic acceleration is able to modify the spectrum of particles accelerated by the shock wave.

Instead of solving the integral exactly, we replace the integrand by its value at the shock and integrate from 0 to $d$. Thus,

$R_{\mathrm{II}, \beta>1} \approx\left(1-H_{\mathrm{c} 0}^{2}\right) \frac{v_{\mathrm{A}}}{v} \frac{(\beta-1)(\sqrt{\beta}+1)\left(M^{2}-1\right)}{24\left(M+H_{\mathrm{c} 0}\right)\left|M H_{\mathrm{c} 0}+1\right|}$,

which is a small number in a large region of parameter space. In the case of low-beta plasma, we obtain in a similar manner

$R_{\mathrm{II}, \beta<1} \approx\left(1-H_{\mathrm{c} 0}^{2}\right) \frac{v_{\mathrm{A}}}{v} \frac{M^{2}-1}{48\left(M+H_{\mathrm{c} 0}\right)\left|M H_{\mathrm{c} 0}+1\right|}$,

if $\mathfrak{E}^{ \pm} \propto k^{-1}$. For other spectral forms of the Alfvén waves, we expect the numerical factors to be different but the qualitative result to be the same.

Thus, if the three-wave processes studied in this paper govern the Alfvén-wave evolution in the downstream region of parallel shocks, stochastic acceleration is typically less important than first-order Fermi acceleration. Thus, unlike in an earlier model of Ko (1998), the plasma seems able to get rid of the bi-directional Alfvén-wave field without having to damp the energy of the minor-wave mode on the accelerated particles. This justifies using power-law particle-energy spectra (Eq. (1)) resulting from the first-order acceleration. Exceptions from this may be strong (or even relativistic) shock waves, for which $\beta$ is very large, as well as some special cases for which the cross-helicity is close to the critical value $H_{\mathrm{c} 0} \approx-M^{-1}$. The scattering-center compression ratio in Eq. (1) needs to be determined either at the shock or in the far downstream region depending on the value of the parameter $R_{\mathrm{I}}$ given by Eq. (83). We note, however, that the description of wave-wave interactions adopted in this paper is very simple, and including other types of wave cascading in the analysis may change the results, even qualitatively.

\section{Conclusions}

We have studied three-wave interactions of Alfvén waves and sound waves in super-Alfvénic, one-dimensional flows, such as those in parallel shocks. Our analysis shows that wave intensity tends toward a degenerate value, $H_{\mathrm{c}} \rightarrow \pm 1$, far from the shock, where the sign is determined by the wave spectra at the shock. Since the cross-helicity of the waves tends toward a degenerate value in a distance that is typically small when compared to the distance required for efficient downstream stochastic acceleration, test-particle acceleration at such shocks is typically governed by the first-order Fermi mechanism. The spectral index of the accelerated particles typically remains close to the values predicted by Vainio \& Schlickeiser (1999), although some parameter regions where modifications are expected were identified.

Acknowledgements. This study is a part of the project "Collective Processes in Astrophysical Plasmas: Waves, Heating and Accelerated Particles" co-funded by the Academy of Finland (Project 204465) and Deutscher Akademischer Austauschdienst (DAAD Project 313-PPP$\mathrm{SF} / 04 / \mathrm{Ik})$.

\section{References}

Axford, W. I., Leer, E., \& Skadron, G. 1977, Proc. 15th Int. Cosmic Ray Conf., 11, 132

Bell, A. R. 1978, MNRAS, 182, 147

Blandford, R. D., \& Ostriker, J. P. 1978, ApJ, 221, L29

Braginskii, S. I. 1965, Rev. Plasma Phys., 1, 205

Campeanu, A., \& Schlickeiser, R. 1992, A\&A, 263, 413

Chin, Y., \& Wentzel, D. G. 1972, Ap\&SS, 16, 465

Krymsky, G. F. 1977, Sov. Phys. Dokl., 22, 327

Ko, J.-M. 1998, A\&A, 304, 605

McKenzie, J. F., \& Westphal, K. O. 1969, Planet. Space Sci., 17, 1029

Ostrowski, M., \& Schlickeiser, R. 1993, A\&A, 268, 812

Ostrowski, M., \& Schlickeiser, R. 1996, Sol. Phys., 167, 381

Schlickeiser, R. 1989a, ApJ, 336, 243

Schlickeiser, R. 1989b, ApJ, 336, 264

Schlickeiser, R., Campeanu, A., \& Lerche, I. 1993, A\&A, 276, 614

Sagdeev, R. Z., \& Galeev, A. A. 1969, Nonlinear Plasma Theory (New York: Benjamin), 1969

Skilling, J. 1975a, MNRAS, 172, 557

Skilling, J. 1975b, MNRAS, 173, 245

Skilling, J. 1975c, MNRAS, 173, 255

Vainio, R., \& Schlickeiser, R. 1998, A\&A, 331, 793

Vainio, R., \& Schlickeiser, R. 1999, A\&A, 343, 303

Vainio, R., Kocharov, L., \& Laitinen, T. 2000, ApJ, 528, 1015

Wentzel, D. G. 1974, Sol. Phys., 39, 129 